\title{
Lethal synergistic action of toxins of bacteria isolated from sudden infant death syndrome
}

\author{
D B Drucker, H S Aluyi, J A Morris, D R Telford, A Gibbs
}

\begin{abstract}
Aim: To test the hypothesis that lethal toxins of bacteria associated with sudden infant death syndrome (SIDS) can act synergistically.

Methods: Bacteria occurring together in the nasopharynx of cases of cot death were studied. The lethal toxicity of crude toxin preparations was determined over a range of dilutions by injections into the chorioallantoic vein of the chick embryo. Toxin preparations of low lethality for the chick embryo SIDS model were then tested in combination.

Results: Staphylococcus aureus toxin preparations showed low lethality when tested alone, even at low dilution. At 1 in 100 dilution $S$ aureus toxin was lethal to one out of 15 chick embryos. Escherichia coli toxin preparations showed high lethality except on high dilution ( 1 in 80) when lethality fell to two out of 15 of chick embryos. When the same toxin preparations were tested simultaneously in combination, lethality rose to 14 out of 15 . Similar findings were observed over a range of toxin dilutions. This finding was highly significant $(p=0.0012)$.

Conclusions: That synergy between toxins can enhance the lethality of toxins elaborated by bacteria associated with SIDS.
\end{abstract}

In England and Wales, the major cause of death of infants aged 1 week to 1 year is cot death, also known as sudden infant death syndrome (SIDS). The latest published figures reveal that in 1990 SIDS claimed 1079 infants. ${ }^{1}$ Diagnosis of SIDS is made by exclusion of other causes of death. Various theories of SIDS aetiology have been reviewed elsewhere. ${ }^{2}$ Many of the hypotheses advanced have not addressed the age distribution of SIDS which shows a peak at about 3 months of age. One hypothesis which can explain the observed age distribution in SIDS is the "common bacterial toxin hypothesis". ${ }^{3}$ This permits theoretical computation of an age incidence very close to the actual observed data. The hypothesis proposes that SIDS occurs when an infant is exposed to a commonly occurring toxigenic micro-organism to which it lacks immunity. At about 3 months, a baby's maternal immunoglobulins have declined whereas its actively acquired immunity will not be fully effective. Others have proposed botulin as a candidate SIDS toxin. ${ }^{45}$ Botulin is an exceptionally potent neurotoxin but is elaborated by only one bacterial species-Clostridium botulinum. The latter is rarely a cause of human food poisoning, let alone death, in England and Wales.

The suggestion that SIDS may result from fatal toxaemia due to commonly occurring bacteria is supported by several studies. An epidemiological study ${ }^{6}$ has found that SIDS babies have an altered nasopharyngeal flora compared with matched healthy infants. The relevance of the nasopharyngeal flora is that SIDS cases frequently have upper respiratory tract infection ${ }^{7}$ which predisposes to bacterial proliferation. A second study has shown that SIDS cases are significantly more likely than matched healthy infants to harbour a lethally toxigenic micro-organism in their nasopharynx. The role of bacterial toxins in SIDS may be enhanced by other agents. ${ }^{8}$ Two recent studies ${ }^{910}$ have shown that, using the ferret as an experimental model, influenza virus can enhance the actions of staphylococcal $\alpha$ toxin, diphtheria toxin, and streptolysin S.

Using the gnotobiotic rat as a model for SIDS, it has previously been shown that infecting bacteria isolated from SIDS, such as $E$ coli and $S$ aureus, can act in combination to produce sudden death ${ }^{11}$ whereas neither organism is able to separately. However, the "common bacterial toxin hypothesis" suggests SIDS is the result of bacterial toxins rather than infecting bacteria per se. Thus the aim of this study is to determine whether bacterial cell-free toxin preparations from different bacteria isolated from the nasopharynx of a SIDS case might show lethal synergy.

\section{Methods}

The strains used in this study were Escherichia coli SIDS-04, and Staphylococcus aureus SIDS03. Both were isolated from the nasopharynx of a single SIDS case, a 15 week old boy who died in September. He had $S$ aureus, $K$ aerogenes, $E$ coli and $C$ albicans isolated from his nasopharyngeal flora. The sampling, isolation, and speciation of the organisms has been described elsewhere. ${ }^{8}$

To prevent contamination of samples by environmental endotoxin, all glassware was washed in E-Toxa (Sigma, Poole), rinsed in pyrogen-free water and sterilised at $200^{\circ} \mathrm{C}$ for five hours in a hot air oven. Test organisms were grown in flasks of buffered salt solution; HBSS (Sigma, Poole) with brain-heart infusion, BHI (Oxoid, Basingstoke). The medium was prepared by dialysing $100 \mathrm{ml} \mathrm{BHI} \mathrm{(37} \mathrm{g/l)}$ against $100 \mathrm{ml}$ HBSS, which was pyrogen-free 
and sterile, overnight at $37^{\circ} \mathrm{C}$. The HBSS-BHI dialysate was inoculated with test organism using $20 \mu \mathrm{l}$ of early stationary phase cultures. After incubating at $37^{\circ} \mathrm{C}$ for 20 hours, the optical density was determined at $\lambda 550 \mathrm{~nm}$, with the aid of a double beam spectrophotometer (Pye-Unicam, Cambridge). To ensure that photometric error was minimised, appropriate dilutions of culture were used for absorbance measurement and a notional value calculated for undiluted culture. Cells were removed by centrifugation at $3000 \times g$ for 15 minutes, followed by filtration of supernatant fluid through a $0.20 \mu \mathrm{M}$ pore size membrane (Gelman Sciences) to remove any residual cells. The cell-free filtrate was diluted so that crude toxin preparations were derived from cultures of standard optical density (absorbance), as previously described. ${ }^{8}$ Preparations were freeze dried and stored at $-20^{\circ} \mathrm{C}$.

Whole crude toxin preparations were tested because in vivo bacteria elaborate a range of extracellular products rather than a pure exo-, or endotoxin. The chick embryo test system has been described elsewhere ${ }^{8}$ and is based on the technique of Eichhorn. ${ }^{12}$ Briefly, toxin samples $(50 \mu \mathrm{l})$ were injected into the chorioallantoic vein of 11 day old chick embryos which were examined after 24 hours. Occasionally injection resulted in haemorrhage, in which case replacement embryos were used. Only healthy chick embryos, which were active on candling, were used.

Three series of experiments were performed. Firstly, a preliminary series of dilutions of toxin preparations were tested to discover appropriate dilutions for synergy testing. $E$ coli toxin preparations were tested at the following dilutions: undiluted stock, 1 in 2,1 in 5,1 in 8 , 1 in 10,1 in 15,1 in 20,1 in 25,1 in 30,1 in 35,1 in 40,1 in 45, 1 in 50,1 in 80 and 1 in 100. $S$ aureus toxin preparation was tested undiluted and at 1 in 2, 1 in 5, 1 in 10, 1 in 20, 1 in 40 and 1 in 100 dilutions. A total of 187 chick embryos was used for this series of tests. A second preliminary series of experiments used five chick embryos per toxin permutation to assay all possible permutations of $E$ coli toxin ( 1 in 10,1 in 20,1 in 40,1 in 80,1 in 100) and $S$ aureus toxin ( 1 in 2,1 in 5,1 in 10,1 in 20, 1 in 40,1 in 100); undiluted $S$ aureus and $E$ coli toxins were also tested and a negative control of HBSS was included. A third series of experiments used fewer permutations of toxin
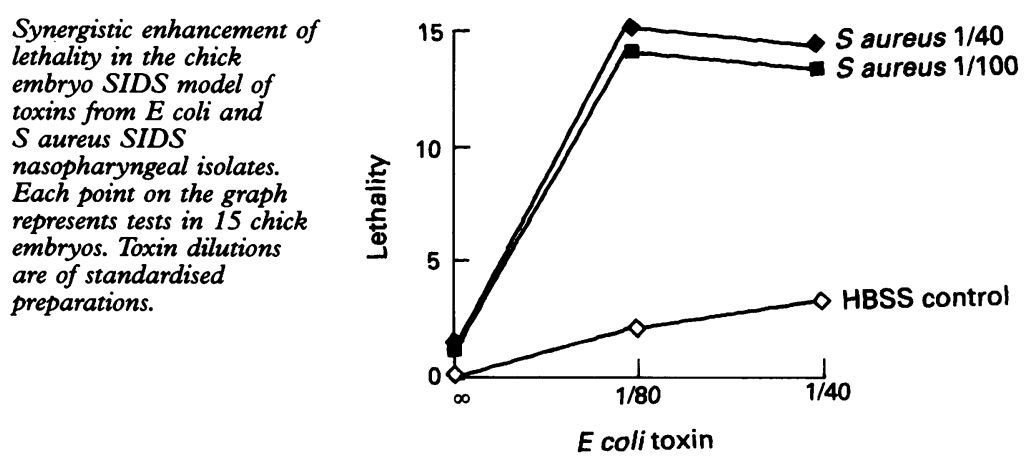

Table Enhancement of lethality of $E$ coli toxin preparations by $S$ aureus toxin preparation

\begin{tabular}{llrr}
\hline \multirow{2}{*}{$S$ aureus dilutions } & \multicolumn{3}{l}{ E coli dilutions } \\
\cline { 2 - 4 }${ }^{*} \infty$ & 1 in 80 & 1 in 40 \\
\hline$\star_{\infty}$ & $0 / 15$ & $2 / 15$ & $3 / 15$ \\
$1 / 100$ & $1 / 15$ & $14 / 15$ & $13 / 15$ \\
$1 / 40$ & $1 / 15$ & $15 / 15$ & $14 / 15$ \\
$1 / 20$ & $2 / 15$ & $12 / 15$ & $10 / 15$ \\
$1 / 2$ & $4 / 15$ & $14 / 15$ & $15 / 15$ \\
\hline
\end{tabular}

${ }^{\star} \infty=$ infinite dilution-toxin free negative control. Significance: $p=0.0012$.

dilutions but tested each sample in 15 chick embryos to permit subsequent statistical analysis of data. This last series of experiments used $E$ coli toxin (1 in 40,1 in 80) $S$ aureus toxin (1 in 2, 1 in 20,1 in 40,1 in 100) and HBSS to replace $E$ coli and/or $S$ aureus toxin preparations. Thus 15 permutations were tested, each in 15 chick embryos. After reading results the possibility of death resulting from bacterial infection was examined by bacterial culture of material from chick embryos which were no longer viable.

\section{Results}

The first series of experiments revealed that undiluted $E$ coli toxin was lethal to $100 \%$ of chick embryo tested and even 1 in 25 dilution killed $80 \%$ of chick embryos; however, 1 in 45 dilution was lethal to only $17 \%$ of chick embryos tested. $S$ aureus, on the other hand, proved relatively non-lethal in the test system. Undiluted toxin preparation killed only $20 \%$ of chick embryos. At dilutions greater than 1 in 10 , no chick embryos were killed.

In the second series of experiments, there seemed to be synergy when $E$ coli and $S$ aureus toxins preparations were tested in combination. For example, $E$ coli at 1 in 100 and 1 in 80 dilution without $S$ aureus toxin each killed one out of five chick embryos. These figures rose to four and five out of five, respectively, in combination with $S$ aureus toxin at 1 in 40 dilution. The latter on its own was non-lethal. The third series of experiments, using greater numbers of chick embryos per toxin sample yielded the data presented in the table. These data appear even more striking when plotted graphically (figure). Clearly, the potency of toxin derived from $E$ coli is greatly enhanced by $S$ aureus toxin preparations. The increase in lethality is far greater than can be explained as an additive effect, so that synergy must have occurred.

The data were analysed statistically to determine the significance of these findings using log linear models. ${ }^{13}$ The programme Hiloglinear from the SPSS package was used to investigate the correlation between lethality and the dilution concentrations of $E$ coli and $S$ aureus. ${ }^{14}$ The effect of the interaction of the different concentrations of $E$ coli and $S$ aureus on lethality was tested using a likelihood ratio test based on the $\chi^{2}$ distribution with 8 degrees of freedom. The result was significant; $\chi_{8}^{2}=25 \cdot 70 ; p=0.0012$. 


\section{Discussion}

The finding that $S$ aureus toxin is less lethal to chick embryos than $E$ coli toxin parallels the authors' recent experience with viable strains. ${ }^{8}$ $E$ coli has also been associated with SIDS cases in a comparison of SIDS and matched healthy babies ${ }^{6}$ and in an examination of toxigenic $E$ coli strains. ${ }^{15}$ Live infective organisms, rather than toxin preparations, have yielded very similar findings in the germ-free rat model of SIDS. ${ }^{11}$ In the latter study $S$ aureus produced only localised infections. $E$ coli alone produced death after septicaemia, 48 hours after infection; $S$ aureus plus $E$ coli resulted in sudden death following a rapid onset of illness. The fact that similar conclusions can be reached from use of very different SIDS models lends particular weight to the importance of synergy between toxins in SIDS. Further evidence of possible enhancement of a toxin's lethality comes from studies using influenza virus plus bacterial toxins in ferret. ${ }^{910}$ It is conceivable that prior viral infection, followed by nasopharyngeal mixed bacterial secondary infection, could yield even greater enhancement of lethality. The present findings further support the common bacterial toxin hypothesis ${ }^{3}$ and suggest a rationale to be explored for future prevention of SIDS. For example, elimination of enterobacteria from the nasopharynx might greatly reduce the lethality of toxins from other bacteria in infants at risk from SIDS.

Further work is in progress to characterise the toxins elaborated by the strains examined in this study.
We thank the Foundation for the Study of Infant Death for their support.

1 Foundation for the Study of Infant Deaths. Cot death research and support. Newsletter 1991;40:2.

2 Milner AD. Recent theories on the cause of cot deaths. $\mathrm{Br}$ Med F 1987;295:1366-8.

3 Morris IA, Haran D, Smith A. Hypothesis: common bacterial toxins are possible cause of sudden infant death syndrome. Med Hypoth 1987;22:211-22.

4 Sonnabend OAR, Sonnabend WFF, Krech U, Molz G Sigrist T. Continuous microbiological and pathological study of 70 sudden and unexpected infant deaths: toxigenic intestinal Clostridium botulinum infection in 9 cases of sudden infant death syndrome. Lancet 1985; $237-41$.

5 Arnon SS, Midura TF, Damus K, Wood RM, Chin J. Intestinal infection and toxin production by Clostridium botulinum as one cause of sudden infant death syndrome. Rev Infect Dis 1978;6:S193-S201.

6 Telford DR, Morris JA, Hughes P, et al. The nasopharyngeal bacterial flora in the sudden infant death syndrome. $\mathcal{F}$ bacterial flora in the

7 Williams AL, Uren CE, Bretherton L. Respiratory viruses and sudden infant death. Br Med $\mathcal{F}$ 1984;288:1491-3.

8 McKendrick N, Drucker DB, Morris JA, et al. Bacterial toxins: a possible cause of cot-death. $f$ Clin Patho 1992;45:49-53.

9 Jakeman KJ, Rushton DI, Smith H, Sweet C. Exacerbation of bacterial toxicity to infant ferrets by influenza virus: possible role in sudden infant death. $\mathcal{F}$ Infect Dis 1991;163:35-40.

10 Jakeman KJ, Smith $H$, Sweet $C$. Influenza virus enhancement of membrane leakiness induced by staphylococcal $\alpha$-toxin diphtheria toxin and streptolysin S. $\mathcal{f}$ Gen Virol 1991;72:111-5

11 Lee S, Barson AJ, Drucker DB, Morris JA, Telford DR Lethal challenge of gnotobiotic weanling rats with bacteLethal challenge of gnotobiotic weanling rats with bacte(SIDS). 7 Clin Pathol 1987;40:393-6.

12 Eichhorn EA. A technique for the intravenous inoculation of chick embryos. Science 1940;92:245-6.

13 Bishop Y, Fienberg P, Holland P. Discrete multivariate analysis. Cambridge, Ma: MIT Press, 1975.

14 Norusis MJ. SPSS-X Advanced statistics guide. Chicago SPSS Inc, 1988.

15 Bettleheim KA, Goldwater PN, Dwyer BW, Bourne AJ, Smith DL. Toxigenic Escherichia coli associated with sudden infant death syndrome. Scand f Infect Dis 1990; 22:467-76. 